\title{
Immune cell migration in inflammation: present and future therapeutic targets
}

\author{
Andrew D Luster ${ }^{1}$, Ronen Alon ${ }^{2}$ \& Ulrich $\mathrm{H}$ von Andrian ${ }^{3}$
}

The burgeoning field of leukocyte trafficking has created new and exciting opportunities in the clinic. Trafficking signals are being defined that finely control the movement of distinct subsets of immune cells into and out of specific tissues. Because the accumulation of leukocytes in tissues contributes to a wide variety of diseases, these "molecular codes' have provided new targets for inhibiting tissue-specific inflammation, which have been confirmed in the clinic. However, immune cell migration is also critically important for the delivery of protective immune responses to tissues. Thus, the challenge for the future will be to identify the trafficking molecules that will most specifically inhibit the key subsets of cells that drive disease processes without affecting the migration and function of leukocytes required for protective immunity.

The past three decades have witnessed an explosion of knowledge in immunology, which is being increasingly 'translated' into new therapies for seemingly unrelated human pathologies caused by excessive or misdirected inflammatory responses. Although such diseases can affect any part of the body, almost all inflammatory conditions are restricted to particular target organs or tissue components ${ }^{1-3}$. Examples include (among many others) inflammatory diseases affecting the skin (psoriasis and eczema), intestine (Crohn disease and ulcerative colitis) or central nervous system (multiple sclerosis and Alzheimer disease); rheumatoid arthritis; asthma; arteriosclerosis; and juvenile diabetes. This focused pathology indicates a critical function for specific tissue infiltration by distinct effector leukocytes in inflammatory disease pathogenesis. Thus, inhibition of blood-borne effector cell recruitment should provide a means for effective and selective anti-inflammatory therapy ${ }^{1-3}$. Extensive preclinical studies provide strong support for this idea, and clinical developments have indicated that pharmacological inhibitors of leukocyte migration can be highly effective in certain autoimmune conditions.

This review summarizes the understanding of leukocyte trafficking with particular emphasis on the following questions: What leukocyte subsets are linked to inflammatory diseases? How and where are they generated and how do they induce disease? What molecular tools do

\footnotetext{
${ }^{1}$ Center for Immunology and Inflammatory Diseases, Division of Rheumatology, Allergy and Immunology, Department of Medicine, Massachusetts General Hospital, Harvard Medical School, Boston, Massachusetts 02114, USA. 2Department of Immunology, Weizmann Institute of Science, Rehovot 76100, Israel. ${ }^{3}$ The CBR Institute for Biomedical Research and Department of Pathology, Harvard Medical School, Boston, Massachusetts 02115, USA. Correspondence should be addressed to A.D.L. (Iuster.andrew@mgh.harvard.edu), R.A. (ronen.alon@weizmann.ac.il) or U.H.v.A. (uva@cbr.med.harvard.edu).

Published online 17 November 2005; doi:10.1038/ni1275
}

they deploy to migrate to target tissues? How do trafficking molecules regulate effector cell recruitment in vessels and during subsequent transendothelial and interstitial migration? Which trafficking molecules are promising targets for safe and effective drug inhibition? Which migration-directed drugs might be effective in which disease(s) and why? What are the risks and benefits of antimigration therapy?

\section{The main cellular participants}

'Newly minted' leukocytes that enter the blood from the bone marrow (innate immune cells and B cells) or thymus (naive T cells) express characteristic trafficking molecule patterns that enable and restrict their migration to certain regions. In general, innate immune cells respond to inflammation-induced traffic cues, whereas naive lymphocytes are poorly responsive to inflammatory signals but migrate efficiently to secondary lymphoid tissues. All leukocytes can respond to activation signals by altering the composition, expression and/or functional activity of their trafficking molecules. However, whereas these changes are relatively uniform in most innate immune cells, the migratory patterns acquired by activated B cells and T cells are diverse and depend on the quality, strength and context of the antigenic stimulus. After being activated, different effector leukocyte subsets must localize together in affected tissues to communicate through short-range cytokines and/or direct cell-cell contact. Antimigration therapy interferes with the pathological gathering of these effector cells. Some diseases can be treated by blocking one essential traffic signal for the recruitment of a pathogenic subset(s), whereas others involve mixed subsets that can use different traffic signals to accumulate in the same target tissue, thus necessitating combination therapy for maximal benefit.

Granulocytes, which include neutrophils, eosinophils and basophils, are innate immune cells that contain specialized granules to provide the first line of immune defense. Neutrophils are the most abundant bloodborne leukocytes in healthy humans. These short-lived cells accumulate 


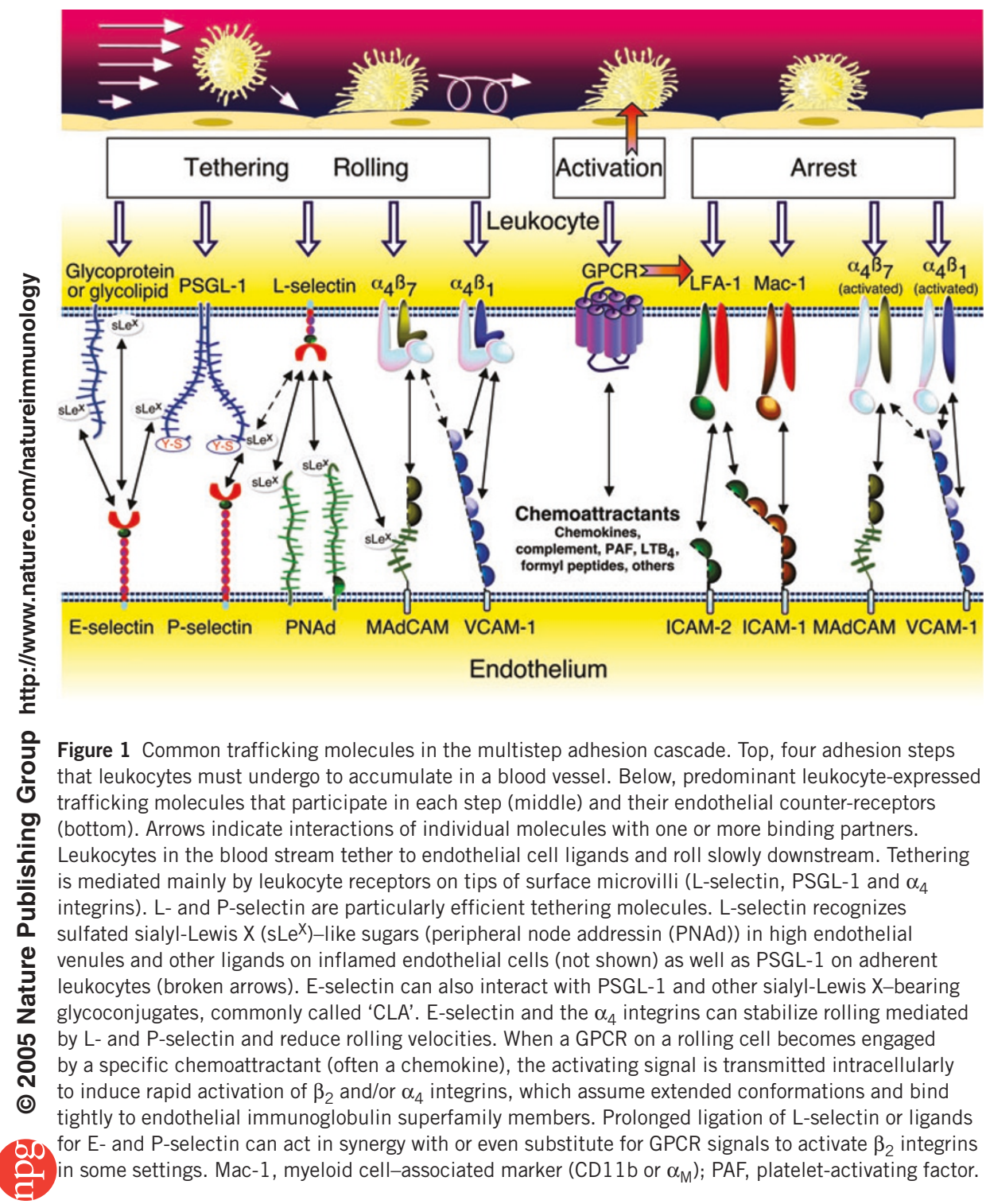

express a broad range of adhesion molecules and chemoattractant receptors; different subsets can be distinguished in mice based on their trafficking molecules and differential precursor potential for tissue-resident leukocytes.

DCs are rare in peripheral blood but are abundant in lymphoid and certain nonlymphoid tissues. Several subsets exist in mice and humans with distinct immunological activities, tissue distribution and migratory properties. This includes conventional myeloid DCs and plasmacytoid DCs, the main interferon- $\alpha$ producing DC subset ${ }^{4}$. DCs are the quintessential 'professional' antigen-presenting cells ${ }^{5}$ and are critical for priming $\mathrm{CD} 4^{+}$and $\mathrm{CD} 8^{+} \mathrm{T}$ cells against exogenous cell-associated antigens ${ }^{6}$. They collect and process antigenic material and, in response to maturation signals, migrate to lymphoid tissues to trigger $\mathrm{T}$ cell activation. Drugs that interfere with DC maturation or migration may be useful for blocking immune responses to neoantigens, whereas deliberate boosting of DC migration could aid vaccine development.

Mast cells are the prototypical tissueresident sentinels. Bone marrow-derived mast cell precursors take up residence in peripheral tissues, often in close contact with arterioles and venules. Inflammatory signals such as tissue damage, infections or crosslinked immunoglobulin E induce mast cell degranulation and release of cytokines, chemokines and 'secretagogues', which enhance venular permeability and leukocyte recruitment. Although it is uncertain whether inhibitors of mast cell trafficking would have therapeutic benefits, suppression of mast cell activation or function can indirectly interfere with mast cell-induced recruitment of other inflammatory cells.

Lymphocytes provide antigen-specific acquired immunity and immunological mem-

within hours at sites of acute inflammation. Neutrophils express abundant adhesion molecules for rapid binding to inflammation-induced counter-receptors on activated endothelial cells, and their chemoattractant receptors sense the release of tissue 'distress signals' and pathogen-derived or pathogen-induced molecules. Neutrophils are essential for combating bacterial and fungal infections, but their activation also releases cytototoxic mediators, causing tissue damage. Neutrophils are often prominent components of $\mathrm{T}$ helper type $1\left(\mathrm{~T}_{\mathrm{H}} 1\right)$-associated inflammatory infiltrates. In contrast, the less abundant eosinophil is typically found in $\mathrm{T}_{\mathrm{H}} 2$ lesions, including sites of parasitic infections and allergic inflammation. Eosinophils express distinct trafficking molecules, particularly receptors for certain chemokines that are selectively induced by $\mathrm{T}_{\mathrm{H}} 2$ cytokines.

Monocytes, unlike granulocytes, are long lived and can differentiate to become tissue-resident macrophages or dendritic cells (DCs). Monocytoid cells have been linked to many inflammatory diseases, particularly arteriosclerosis, in which monocytes accumulate in atherosclerotic plaques and turn into lipid-laden foam cells. However, monocytes also provide essential beneficial effects, such as during wound healing and to combat or contain chronic bacterial infections. Monocytes ory and include many subsets of $\mathrm{T}$ cells and $\mathrm{B}$ cells with distinct trafficking patterns. Although naive T cell and B cell trafficking is restricted mainly to lymphoid tissues, effector and memory lymphocytes adapt their migratory 'preferences' to match their immunological function. So far, activation-induced changes in effector cell migration have been best explored in $\mathrm{T}$ cells ${ }^{7}$, but similar rules also apply to antibody-secreting $\mathrm{B}$ cells $\mathrm{s}^{8}$. The trafficking properties of activated $\mathrm{T}$ cells are influenced by several factors. Compared with naive $\mathrm{T}$ cells, antigen-experienced $\mathrm{T}$ cells travel more efficiently to inflamed tissues because they upregulate adhesion molecules and chemoattractant receptors for inflammation-induced ligands ${ }^{9}, 10$. However, there are important additional distinctions between different memory $\mathrm{T}$ cell subsets. First, antigenexperienced $\mathrm{T}$ cells can be subcategorized as central memory cells, which keep recirculating through all lymphoid tissues, and effector memory cells, which lack one or more prerequisite receptors for recirculation ${ }^{11}$. Second, antigen challenge in different lymphoid organs, such as mucosaassociated lymphoid organs or skin-draining lymph nodes, generates preferentially gut- or skin-seeking effector cells, respectively ${ }^{12}$. Tissuespecific 'imprinting' also occurs during the induction of central nervous system-tropic effector cells in cervical lymph nodes ${ }^{13}$. Third, effector 
cell trafficking is influenced by cytokines that polarize $\mathrm{T}_{\mathrm{H}} 1$ and $\mathrm{T}_{\mathrm{H}} 2$ responses ${ }^{14,15}$. Notably, these distinctions are not absolute. For example, tissue-specific memory cells can also express lymph node-homing receptors $^{16}$, and effector cells can revert to a central memory phenotype ${ }^{17}$. Similarly, polarizing cytokines can modulate the magnitude of traffic molecule expression on activated $\mathrm{T}$ cells but cannot override tissuespecific 'imprinting' signals provided by DCs from different lymphoid tissues $^{12}$

There are additional lymphocyte subsets, such as natural killer cells, natural killer $\mathrm{T}$ cells and regulatory $\mathrm{T}$ cells, that are also important in host responses to infection and in regulating inflammation and disease. Regulatory $\mathrm{T}$ cells share trafficking molecules with other leukocytes, so inhibitors may inadvertently target both effector and regulatory subsets, with unforeseen consequences for disease pathology and susceptibility to infection. This must be kept in mind and monitored as new antiadhesion therapies enter the clinic.

\section{The molecular participants: defining the targets}

Adhesion molecules belong mainly to one of three prominent families, with some exceptions (Fig. 1). The selectins comprise a threemember family of highly conserved C-type lectins (Supplementary Table 1 online), which bind sialyl-Lewis X-like carbohydrate ligands presented by sialomucin-like surface molecules such as P-selectin glycoprotein ligand 1 (PSGL-1) ${ }^{18}$. Selectin-mediated adhesion bonds are characterized by high 'on rates' and 'off rates' and last only a few seconds or less. L-selectin is expressed on most circulating leukocytes and is the key receptor that initiates leukocyte capture events in high endothelial venules in secondary lymphoid tissues and at peripheral sites of injury and inflammation, which inducibly express specific sulfated and fucosylated sialoglycoproteins ${ }^{19}$. With few exceptions, P- and E-selectin are inducibly expressed in both acutely and chronically stimulated endothelial beds and are important determinants for neutrophil, monocyte, natural killer cell, eosinophil and effector T cell and B cell recruitment in most inflammatory processes ${ }^{18}$. L-selectin on circulating leukocytes can also bind leukocyte ligands, particularly PSGL-1, and this interaction can enhance the capturing of leukocytes by intravascular adherent leukocytes $^{20}$. Selectin ligand function depends on complex post-translational modifications of scaffold proteins by glycosyl- and sulfotransferases, several of which are inducible and/or expressed in specialized leukocyte and endothelial cell subsets. The adhesive activities of both selectins and their ligands can be also regulated by their topographical distribution and associations with the actin cytoskeleton and also by shedding ${ }^{21}$.

Integrins constitute a family of two dozen heterodimers whose ligandbinding activity can be rapidly regulated by conformational changes as well as by transcriptional induction and redistribution from intracellular pools ${ }^{22}$. The most relevant integrins for leukocyte migration are members of the $\beta_{2}$ subfamily, especially LFA-1 (CD11a-CD18 or $\alpha_{L} \beta_{2}$ ), and the two $\alpha_{4}$ integrins, $\alpha_{4} \beta_{1}$ (VLA-4) and $\alpha_{4} \beta_{7}$. Integrins of the $\beta_{1}$ subfamily, especially VLA-1, VLA-2, VLA-4, VLA-5 and VLA- 6 , have been linked to leukocyte crossing of the basement membrane underlying blood vessels and to interstitial migration on extracellular matrix (ECM) as well as to leukocyte migration across or retention on inflamed stromal and epithelial cells (Supplementary Table 2 online). Integrins bind certain ECM molecules as well as cell surface receptors belonging to the immunoglobulin 'superfamily', such as the intercellular cell adhesion molecules (ICAMs), which are binding partners for $\beta_{2}$ integrins; vascular cell adhesion molecule 1 (VCAM-1), the main ligand for $\alpha_{4} \beta_{1}$; and mucosal addressin cell adhesion molecule 1 (MAdCAM-1), the main ligand for $\alpha_{4} \beta_{7}$. Both the integrins and their binding partners are likely targets for anti-inflammatory drugs, and several inhibitors have already been used in the clinic (discussed below).
Chemoattractants, particularly the chemokine system and the lipidbased chemoattractants and their many $G$ protein-coupled receptors (GPCRs), are by far the most diverse group of trafficking molecules ${ }^{1,23}$ (Supplementary Table 3 online). They trigger intravascular leukocyte adhesion to endothelial cells and also provide essential guidance for interstitial leukocyte migration and positioning in both inflamed and noninflamed tissues. Their molecular diversity and selective action on distinct leukocyte subsets as well as their restricted temporal and spatial patterns of expression provide a key mechanism for 'fine-tuning' cellular immune responses. Given the many checkpoints a leukocyte must navigate through to cross tissue barriers, it can be predicted that only specific combinations of chemoattractants will promote productive recruitment of a leukocyte into a target tissue. Inhibitors of both the GPCR binding sites of specific chemoattracts as well as of their presentation in endothelial and stromal compartments ${ }^{24}$, especially when combined with new antiadhesive drugs, should yield specific migration-attenuating therapies in diverse inflammatory settings. Animal studies have indeed indicated that pharmacological inhibitors of chemoattractant pathways can exert potent and specific anti-inflammatory activity, and clinical trials are underway to test their efficacy in particular human diseases.

\section{The multistep adhesion cascade: the paradigm}

Many in vivo and in vitro studies have established that leukocytes circulating in the blood are recruited to lymphoid organs and to peripheral sites of injury, infection and inflammation by a series of overlapping sequential steps mediated by selectins and integrins ${ }^{25,26}$ (Fig. 2). As each step is conditional for the next, multiple molecular 'choices' at each step provide a large combinatorial diversity. This not only generates high specificity but also provides a multitude of specific therapeutic targets. With the exception of $\mathrm{T}$ cell and $\mathrm{B}$ cell blasts, which express highly adhesive integrins ${ }^{27}$, all circulating leukocytes maintain their integrins in mostly inactive states and must undergo in situ modulation to develop high avidity for their specific endothelial ligands to establish firm shearresistant adhesion on target endothelial sites ${ }^{22}$. This notable transition requires the freely flowing leukocyte at the target site, to undergo a reversible capture step mediated mainly by weak selectin-carbohydrate interactions that give rise to leukocyte rolling ${ }^{18}$. Rolling adhesions can last from seconds to minutes, depending on the type of leukocyte and the target endothelial bed ${ }^{28}$. Integrins can also mediate rolling interactions, which often stabilize L-selectin-mediated capture and rolling ${ }^{29}$. In addition, endothelium-expressed oxidases such as VAP-1 may contribute to stabilization of rolling and subsequent integrin-mediated sticking through modifications of endothelial and leukocyte ligands ${ }^{30}$. Given those findings, it is becoming increasingly evident that highly specific combinations of these receptors determine both the number and duration of endothelial contacts ${ }^{21}$ and thereby control the degree of subsequent activation of integrin-dependent sticking.

Lymphocyte and myeloid cell arrest in venules requires in situ activation of at least one of the four main integrins: VLA- $4, \alpha_{4} \beta_{7}$, Mac- 1 and LFA-1 (Supplementary Table 2 online). The molecular basis of integrin activation underlying leukocyte stoppage (sticking and arrest) on target endothelial sites is beginning to unfold ${ }^{31}$. Overlapping and additive proadhesive properties of these integrins depend on their specific cytoskeletal and transmembrane associations with cytoskeletal adaptor molecules ${ }^{32}$ as well as membrane effectors, such as tetraspanins, CD47, CD98 and CD44 (ref. 33). These effectors regulate both conformational switches of the integrin heterodimers and their ability to microcluster and anchor to the actin cytoskeleton ${ }^{22,34}$. Given their restricted expression and function in different subsets of leukocytes, 'bispecific' monoclonal antibodies directed to one of these integrin partners and to its integrin counterpart may function in a cell type-restricted way, much 
Figure 2 Key migration steps of immune cells at sites of inflammation. Inflammation due to tissue damage or infection induces the release of cytokines (not shown) and inflammatory chemoattractants (red arrowheads) from distressed stromal cells and 'professional' sentinels, such as mast cells and macrophages (not shown). The inflammatory signals induce upregulation of endothelial selectins and immunoglobulin 'superfamily' members, particularly ICAM-1 and/or VCAM-1. Chemoattractants, particularly chemokines, are produced by or translocated across venular endothelial cells (red arrow) and are displayed in the lumen to rolling leukocytes. Those leukocytes that express the appropriate set of trafficking molecules undergo a multistep adhesion cascade (steps 1-3) and then polarize and move by diapedesis across the venular wall (steps 4 and 5). Diapedesis involves transient disassembly of endothelial junctions and penetration through the underlying basement membrane (step 6). Once in the extravascular (interstitial) space, the migrating cell uses different integrins to gain 'footholds' on collagen fibers and other ECM molecules, such as laminin and fibronectin, and on inflammation-induced ICAM-1 on the surface of parenchymal cells (step 7). The migrating

cell receives guidance cues from distinct sets of chemoattractants, particularly chemokines, which may be immobilized on glycosaminoglycans (GAG) that 'decorate' many ECM molecules and stromal cells. Inflammatory signals also induce tissue-resident DCs to undergo maturation. Once DCs process material from damaged tissues and invading pathogens, they upregulate CCR7, which allows them to enter draining lymph vessels that express the CCR7 ligand CCL21 (and CCL19). In lymph nodes (LN), these antigen-loaded mature DCs activate naive T cells and expand pools of effector lymphocytes, which enter the blood and migrate back to the site of inflammation. T cells in tissue also use this CCR7-dependent route to migrate from peripheral sites to draining lymph nodes through afferent lymphatics.

more selectively than the 'monospecific' integrin-blocking monoclonal antibodies or drug antagonists ${ }^{35}$.

New structural and functional data strongly suggest that integrins are bidirectionally activated by both cytoplasmic rearrangements of their subunit tails and the binding of their own extracellular ligands ${ }^{36}$. In vivo and in vitro data in $\mathrm{T}$ cells have indicated that integrin activation by endothelium-presented chemokines occurs within a fraction of a second ${ }^{34}$. Abrupt arrest has also been reported in vitro in neutrophils, monocytes and natural killer cells ${ }^{37,38}$. Only specific combinations of chemokines and GPCRs can activate integrin-dependent arrest under shear flow ${ }^{39}$, a process that indicates involvement of in most cases $G_{i}$ protein signaling to two key GTPases, RhoA and Rap-1 (refs. 31,40). Rolling allows the leukocyte to sort the endothelial target for proper arrays of endothelial chemoattractants and integrin ligands. Neutrophils and effector lymphocytes have been also suggested to use prolonged selectin-mediated rolling interactions to activate their integrins ${ }^{41-43}$. Once arrested, leukocytes can use their integrins to bind blood-borne leukocytes and platelets. Platelet activation may facilitate subsequent leukocyte extravasation through localized deposition of platelet derived enzymes, cytokines and chemoattractants ${ }^{44}$.

\section{Crossing vascular and tissue barriers}

Vascular endothelium forms a nonthrombotic, nonadhesive barrier between the blood and tissue that is impermeable to macromolecules ${ }^{45}$. Inflamed endothelial beds undergo diverse and heterogenous changes in permeability to blood constituents and adhesiveness to leukocytes and platelets. Inflamed vascular cells must 'program' leukocytes to cross them with minimal and transient barrier disruption. The leukocyte GTPases Rap- 1 and RhoA coordinate this process ${ }^{46}$. These GTPases use multiple activators (guanine exchange factors) and 'downstream' effectors, some of which are enriched in specific hematopoietic cells. Different inflammatory signals may use specific combinations of these effectors in distinct subsets of cells ${ }^{31,47}$, increasing the likelihood of designing leukocyte-type specific inhibitors to these GTPases effective in attenuating diapedesis. Rac family GTPases, focal adhesion kinases, and specific protein kinase $\mathrm{C}$ and phosphatidylinositol-3-OH kinase $(\mathrm{PI}(3) \mathrm{K})$ isoforms also promote leukocyte motility over and across endothelial barriers $^{48}$. PI(3)K $\gamma$ directs the directional migration of granulocytes, macrophages and DCs and controls mast cell function in various inflammatory conditions. The idea that this and other $\mathrm{PI}(3) \mathrm{K}$ isoforms such as $\mathrm{PI}(3) \mathrm{K} \delta$ have different trafficking functions in distinct immune cell subsets emphasizes that this family of kinases is a potential target in a variety of inflammatory conditions ${ }^{49}$.

Two routes of leukocyte diapedesis have been noted so far both in vivo and in vitro: a paracellular route that dominates most extravasation processes, and a transcellular route reported in vivo for neutrophils and subsets of activated effector $\mathrm{T}$ cells ${ }^{50}$. Both routes demonstrate a proactive function for both apical and junctional endothelial ICAM-1 and VCAM-1, whose expression is differentially increased at specific sites of inflammation ${ }^{51,52}$. Diapedesis in distinct inflammatory settings also indicates sequential occupancy by the migrating leukocytes of constitutively expressed junctional endothelial ligands such as PECAM-1, CD99 and JAMs ${ }^{53,54}$ (Supplementary Table 4 online). Paracellular (junctional) diapedesis depends on the localized and temporal loss of junctional assemblies and adherens junctions stabilized by transmembrane homophilic proteins, mainly VE-cadherin and specific cytoskeletal linkers such as $\beta$-catenins. In some endothelial beds, such as in the brain and in large arteries, complex assemblies called tight junctions maintain additional barriers for leukocyte diapedesis ${ }^{45}$. Activation of Rho family GTPases and modulation of phosphorylation states of various junctional proteins 

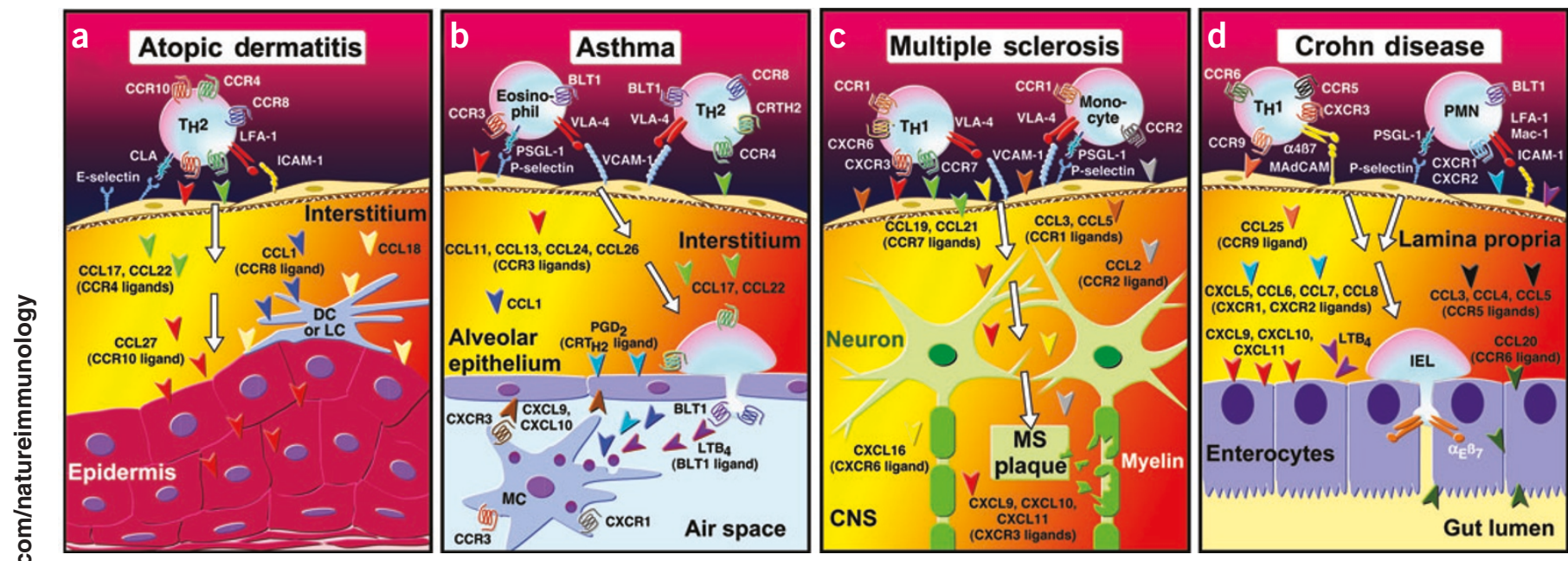

Figure 3 Specific 'combination codes' used by leukocytes in inflammatory diseases. Characteristic $T_{H} 2(\mathbf{a}, \mathbf{b})$ and $T_{H} 1$ (c,d) tissue-restricted pathologies. Atopic dermatitis (a) and asthma (b) are mediated by $\mathrm{T}_{\mathrm{H}} 2$ cells. A comparison of the trafficking molecules that participate in $\mathrm{T}_{\mathrm{H}} 2$ cell recruitment into the skin (a) and the lung (b) shows the tissue- and context-specific nature of the leukocyte recruitment process. Skin-specific homing receptors such as CLA and CCR10 participate in the trafficking of $\mathrm{T}_{\mathrm{H}} 2$ cells to the skin but not to the lung, whereas inflammation-specific homing receptors, such as CCR4 and CCR8, seem to be involved in the trafficking of $\mathrm{T}_{H} 2$ cells to sites of $\mathrm{T}_{H} 2$ inflammation regardless of the inflammatory site. This is also true for the eosinophil, which uses CCR3 and VLA-4 at most sites of allergic inflammation. Likewise, a comparison of the trafficking molecules that participate in $T_{H} 1$ cell recruitment into the brain in multiple sclerosis (c) and the small intestine in Crohn disease (d) also shows the tissue- and context-specific nature of leukocyte trafficking. Gut-specific homing receptors such as $\alpha_{4} \beta_{7}$ and CCR9 participate in the trafficking of TH 1 cells to the small intestine but not to the brain, whereas inflammation-specific homing receptors such as CXCR3 and CCR5 seem to be involved in the trafficking of $T_{H} 1$ cells to sites of $T_{H} 1$ inflammation regardless of the inflammatory site. This is also true for the monocyte, which uses CCR2 at most sites of $T_{H} 1$ inflammation. In atopic dermatitis, CCL27 is expressed mainly by keratinocytes attracting CCR $10^{+} \mathrm{T}_{\mathrm{H}} 2$ cells into the epidermis. Many inflammatory chemokines have been detected in experimental allergic encephalomyelitis, an animal model resembling multiple sclerosis (MS). However, despite the plethora of inflammationinduced changes, the constitutively expressed VCAM-1 (which is further upregulated in multiple sclerosis) and its receptor, VLA-4, have proven to be of particular importance for effector cell recruitment to the brain. LC, leukocyte; $\mathrm{PGD}_{2}$ prostaglandin $\mathrm{D}_{2}$; MC, monocyte; PMN, polymporphonuclear cell; IEL, intraepithelial lymphocyte.

have been linked to paracellular diapedesis; however, these networks maintain blood vessel integrity and would be difficult to target. The use of specific combinations of these endothelial-leukocyte counterreceptor pairs (Supplementary Table 4 online) varies in different diapedesis processes. Both the type of leukocyte and endothelial bed as well as the type and magnitude of the inflammatory stimulus alter the contribution of these counter-receptors to leukocyte diapedesis, suggesting that some of these receptors may be targets for therapeutic inhibition. For example, blockade of PECAM-1 function in several in vivo models attenuates the emigration of specific leukocyte subsets (Supplementary Table 4 online).

Basement membranes and the interstitial ECM of stromal cells are sequentially penetrated by migrating leukocytes transversing endothelial barriers. An emerging theme suggests that engagement of neutrophil PECAM-1 by endothelial PECAM-1 results in upregulation of $\alpha_{6} \beta_{1}$, the main laminin receptor on these leukocytes ${ }^{55}$. PECAM-1 engagement can also potentially activate Rap-1 GTPase on transmigrating leukocytes, resulting in increased avidity of specific $\beta_{1}$ and $\beta_{2}$ integrin receptors for ECM constituents ${ }^{56}$ necessary for leukocyte motility through the basal lamina ${ }^{57}$. Both $\alpha_{6} \beta_{1}$ and the collagenbinding integrins VLA- 1 and VLA-2 are also essential for interstitial locomotion and retention of leukocyte subsets in different inflamed nonlymphoid tissues ${ }^{58}$. It is noteworthy that basement membranes and interstitial ECM are biochemically distinct and vary in composition in lymphoid organs and different tissue type ${ }^{59}$. A notable example of how basal lamina composition determines extravasation profiles is the finding that leukocytes cross blood vessel basement membranes containing laminin type 8 but not those containing type 10 (ref. 60).
The interstitial extracellular matrices are subject to constant remodeling during the inflammatory response, which is associated with increased production of fibronectin, tenascin, fibrillar collagen and distinct sulfated proteoglycans. Distinct types of inflammatory cells may differentially use their membrane-bound and secreted proteases as well as glycosaminoglycan-degrading enzymes to remodel and penetrate ECM barriers, especially while migrating through the tightly interwoven basal lamina ${ }^{61,62}$. Particular proteases are upregulated shortly after transendothelial migration ${ }^{63}$, potentially contributing to basement membrane crossing. Localized proteolysis by migrating leukocytes can also expose cryptic ECM ligands for myeloid cell integrins such as $\alpha_{M} \beta_{2}$ and $\alpha_{x} \beta_{2}$ (ref. 64), modulate chemokine-binding specificity ${ }^{23}$ and release ECM-stored chemokines and inflammatory cytokines involved in leukocyte locomotion and retention ${ }^{65}$. Although they represent likely targets ${ }^{61,66}$, the apparent ubiquity and redundancy of proteases and glycosaminoglycan-degrading enzymes in these processes raises doubts about their utility as targets for therapeutic intervention. It is also noteworthy that lymphocytes, monocytes and probably other leukocyte subsets can navigate in an ameboid way through interstitial ECM independently of protease activities ${ }^{67}$.

\section{Traffic molecules as anti-inflammatory drug targets}

The validity of the multistep paradigm was appreciated with the realization that patients with leukocyte adhesion deficiency syndrome have impaired leukocyte migration into tissues, resulting in recurrent bacterial and fungal infections and a peripheral leukocytosis because of defects at different steps in the multistep paradigm of leukocyte trafficking, including deficient expression of $\beta_{2}$ integrins or fucosylated selectin ligands or an impaired ability to activate leukocyte integrins after chemokine stimulation ${ }^{68}$. Thus, 'experiments of nature' have provided 
Table 1 Trafficking molecules involved in inflammatory disease processes

\begin{tabular}{|c|c|c|c|c|}
\hline \multirow[t]{2}{*}{ Disease } & \multirow[t]{2}{*}{ Key effector cell } & \multicolumn{3}{|c|}{ Proposed leukocyte receptors for endothelial traffic signals } \\
\hline & & L-selectin, ligand & GPCR & Integrin ${ }^{a}$ \\
\hline \multicolumn{5}{|l|}{ Acute inflammation } \\
\hline Myocardial infarction & Neutrophil & PSGL-1 & CXCR1, CXCR2, PAFR, BLT1 & LFA-1, Mac-1 \\
\hline Stroke & Neutrophil & L-selectin, PSGL-1 & CXCR1, CXCR2, PAFR, BLT1 & LFA-1, Mac-1 \\
\hline Ischemia-reperfusion & Neutrophil & PSGL-1 & CXCR1, CXCR2, PAFR, BLT1 & LFA-1, Mac-1 \\
\hline $\mathrm{T}_{\mathrm{H}} \mathbf{1}$ & & & & \\
\hline \multirow[t]{2}{*}{ Atherosclerosis } & Monocyte & PSGL-1 & $\begin{array}{l}\text { CCR1, CCR2, BLT1, CXCR2, } \\
\text { CX3CR1 }\end{array}$ & VLA-4 \\
\hline & $\mathrm{T}_{\mathrm{H}} 1$ & PSGL-1 & CXCR3, CCR5 & VLA-4 \\
\hline \multirow[t]{2}{*}{ Multiple sclerosis } & $\mathrm{T}_{\mathrm{H}} 1$ & PSGL-1 (?) & CXCR3, CXCR6 & VLA-4, LFA-1 \\
\hline & Monocyte & PSGL-1 (?) & CCR2, CCR1 & VLA-4, LFA-1 \\
\hline \multirow[t]{3}{*}{ Rheumatoid arthritis } & Monocyte & PSGL-1 & CCR1, CCR2 & VLA-1, VLA-2, VLA-4, LFA-1 \\
\hline & $\mathrm{T}_{H} 1$ & PSGL-1 & CXCR3, CXCR6 & VLA-1, VLA-2, VLA-4, LFA-1 \\
\hline & Neutrophil & L-selectin, PSGL-1 & CXCR2, BLT1 & LFA-1 ${ }^{\mathrm{b}}$ \\
\hline Psoriasis & Skin-homing $T_{H} 1$ & CLA & CCR4, CCR10, CXCR3 & VLA-4c , LFA-1 \\
\hline Crohn disease & Gut-homing $T_{H} 1$ & PSGL-1 & CCR9, CXCR3 & $\alpha_{4} \beta_{7}$, LFA-1 \\
\hline \multirow[t]{2}{*}{ Type I diabetes } & $\mathrm{T}_{\mathrm{H}} 1$ & PSGL-1 (?) & CCR4, CCR5 & VLA-4, LFA-1 \\
\hline & $\mathrm{CD} 8$ & L-selectin (?), PSGL-1 (?) & CXCR3 & VLA-4, LFA-1 \\
\hline \multirow[t]{2}{*}{ Allograft rejection } & $\mathrm{CD} 8$ & PSGL-1 & CXCR3, CX3CR1, BLT1 & VLA-4, LFA-1 \\
\hline & B cell & L-selectin, PSGL-1 & CXCR5, CXCR4 & VLA-4, LFA-1 \\
\hline Hepatitis & $\mathrm{CD} 8$ & PSGL-1 & CXCR3, CCR5, CXCR6 & VLA-4 \\
\hline \multirow[t]{3}{*}{ Lupus } & $\mathrm{T}_{\mathrm{H}} 1$ & None & CXCR6 & VLA-4 $4^{d}$ \\
\hline & Plasmacytoid DC & L-selectin, CLA & CCR7, CXCR3, ChemR23 & LFA-1, Mac-1 \\
\hline & B cell & CLA (?) & CXCR5, CXCR4 & LFA-1 \\
\hline \multicolumn{5}{|l|}{$\mathrm{T}_{\mathrm{H}} 2$} \\
\hline \multirow[t]{3}{*}{ Asthma } & $\mathrm{T}_{\mathrm{H}} 2$ & PSGL-1 & CCR4, CCR8, BLT1 & LFA-1 \\
\hline & Eosinophils & PSGL-1 & CCR3, PAFR, BLT1 & VLA-4, LFA-1 \\
\hline & Mast cells & PSGL-1 & CCR2, CCR3, BLT1 & VLA-4, LFA-1 \\
\hline Atopic dermatitis & Skin-homing $T_{H} 2$ & CLA & CCR4, CCR10 & VLA-4, LFA-1 \\
\hline
\end{tabular}

aVarious $\beta_{1}$ integrins have been linked in different ways in basal lamina and interstitial migration of distinct cell types and inflammatory settings. ${ }^{\text {ln }}$ some settings, Mac-1 has been linked to transmigration. ${ }^{c} \mathrm{CD} 44$ can act in concert with VLA-4 in particular models of leukocyte arrest ${ }^{33} .{ }^{\mathrm{d}} \mathrm{T}_{\mathrm{H}} 2$ cells require VAP-1 to traffic to inflamed liver.

'proof of principle' in humans that trafficking molecules involved in distinct steps of the multistep trafficking paradigm contribute in unique ways to leukocyte trafficking in vivo. Natalizumab, a monoclonal antibody to the $\alpha_{4}$ integrin chain that blocks the binding of $\alpha_{4} \beta_{1}$ (VLA4) to VCAM-1 on brain-infiltrating $T_{\mathrm{H}} 1$ cells and binding of $\alpha_{4} \beta_{7}$ to MadCAM-1 on gut-infiltrating $\mathrm{T}_{\mathrm{H}} 1$ cells, has been used successfully for the treatment of multiple sclerosis and Crohn disease. This clinical success has established the 'proof of principle' that specifically interfering with leukocyte trafficking into tissue is an effective new therapeutic strategy. However, the specific infectious disease complications that led to the withdrawal of this promising therapy emphasize the need for greater understanding of the components of host defense mediated by specific leukocyte subsets and specific trafficking molecules as well as a need for more precise and specific inhibitors of leukocyte subset trafficking. In this section, we will review the specific types of inflammatory processes and comment on how distinct anatomic compartments influence the trafficking molecules involved in a given disease process (Fig. 3 and Table 1). Finally, we will emphasize promising molecular targets that mediate these processes and then deal with the issue of how it may be possible to avoid infectious complications when inhibiting leukocyte entry into tissue.

The host response to acute infection or injury consists of an exuberant neutrophilic inflammatory response. As is often the case in inflammation, this is a 'double-edge sword', and this robust protective response can also be deleterious to host tissue. The host response to many acute tissue insults such as ischemia followed by reperfusion induces a neutrophil-rich inflammatory response that contributes substantially to tissue injury. Ischemia-reperfusion injury is thought to contribute to many important pathological conditions, including acute myocardial infarction, stroke, shock and acute respiratory distress syndrome. Therapies aimed at blocking neutrophil influx into tissue are being evaluated as likely new strategies for these important disorders. Preclinical 
studies in animal models of ischemia-reperfusion disorders have demonstrated considerable protection against reperfusion injury with blockade of neutrophil selectins, integrins or chemokine receptors ${ }^{2}$. However, clinical trials designed to evaluate the efficacy of inhibiting neutrophil trafficking for ischemia-reperfusion injury have so far been disappointing. For example, trials testing the efficacy of blocking $\beta_{2}$ integrins in the setting of acute myocardial infarction, stroke or traumatic shock have not shown any clinical benefit, and an antibody to ICAM-1, the main counter-receptor for CD18 on endothelial cells, even had a negative effect on strokes in a phase II trial ${ }^{3}$. The reason for the disparity between the preclinical and clinical data is not entirely clear but may relate to the timing or efficacy of inhibition in the human trials. Preclinical studies using blockade of the chief neutrophil chemokine receptors CXCR1 and CXCR2 have also shown efficacy in inhibiting neutrophil influx and tissue damage in models of ischemia-reperfusion injury and are being evaluated in the clinic ${ }^{69-71}$.

$\mathrm{T}_{\mathrm{H}} 1$ inflammation is characterized by tissue infiltration of interferon$\gamma$-secreting $\mathrm{CD}^{+}$and $\mathrm{CD} 8^{+}$effector T cells and activated macrophages. This type of cell-mediated inflammatory response is thought to have an important pathophysiological function in a multitude of prominent human diseases, including type 1 diabetes mellitus, Crohn disease, multiple sclerosis, rheumatoid arthritis, atherosclerosis, psoriasis and solidorgan allograft rejection (Fig. 3). Interfering with trafficking molecules on $\mathrm{T}_{\mathrm{H}} 1$ cells and/or activated macrophages would have the advantage of potentially being beneficial in many of the disorders mentioned above. In fact, inhibition of $\alpha_{L}$ intergrin (CD11a), which together with CD18 forms the LFA-1 complex, has shown promise in the clinic, with the use of Odulimomab for graft-versus-host disease and transplant rejection and Efaluzimab for psoriasis ${ }^{2}$. Furthermore, Natalizumab is very efficacious in Crohn disease ${ }^{72}$ and multiple sclerosis ${ }^{73}$. However, blocking all $\alpha_{4}$-mediated leukocyte trafficking leads to an increased susceptibility to infection. In this case, a small percentage of patients treated with Natalizumab developed progressive multifocal leukoencephalophathy (PML), a deadly opportunistic central nervous system infection caused by reactivation of a clinically latent JC polyomavirus infection. The occurrence of PML in this setting was totally unexpected, as it almost invariably occurs in the setting of profound impaired cell-mediated immunity in patients with AIDS, leukemia or organ transplantation. Therefore, Natalizumab therapy is clearly a 'double-edged sword'. Inhibiting T cell entry into the brain halts the entry of encephalogenic $\mathrm{CD} 4^{+} \mathrm{T}$ cells and the ensuing autoimmune destruction of the myelin sheath. However, inhibiting the entry of another subset of T cells, most likely a subset of $\mathrm{CD}^{+} \mathrm{T}$ cells, leads to reactivation of JC virus. Many issues remain, such as why the JC virus, which lays dormant in $50-80 \%$ of the population, was reactivated in less than $1 \%$ of patients treated with Natalizumab. Furthermore, why did this therapy lead to PML and not other central nervous system opportunistic infections, such as toxoplasmosis?

A greater understanding of the specific T cell subsets that are pathogenic in a given disease and a deeper understanding of the specific infectious diseases held in check by this subset will lead to more effective and better tolerated therapies. Better tools must be developed to specifically inhibit the trafficking of defined subsets of disease-causing cells. This may be possible with therapies aimed at inhibiting organ-specific trafficking molecules. For example, specifically blocking $\alpha_{4} \beta_{7}$-MadCAM interaction and not $\alpha_{4} \beta_{1}$-VCAM-1 interaction should inhibit the entry of $\mathrm{T}$ cells into the gut while not affecting $\mathrm{T}$ cell entry into the central nervous system. In fact, a 'humanized' monoclonal antibody that blocks the $\alpha_{4} \beta_{7}$ heterodimer but not the individual components is effective for the treatment of ulcerative colitis ${ }^{74}$.

Alternatively, organ and immune subset-specific combinatorial therapies should be defined, which will combine partial blockage of selectins, chemokine receptors and integrin members, each contributing to the overall trafficking of the key inflammatory subset underlying the pathology of the particular disease. For example, two individual drugs that selectively inhibit the rolling and arrest step by $75 \%$ each may not be very effective in blocking inflammatory disease when given alone. However, a combination of the two drugs would be expected to reduce effector cell influx by about $94 \%$.

Thus, therapies aimed at blocking the chemokine system, when combined with partial selectin- and integrin-blocking therapy, may afford the needed specificity and efficacy. For example, the CCL25-CCR9 interaction is believed to be important in guiding the entry of $\mathrm{T}$ cells into the small intestine ${ }^{75}$ and therefore represents a likely target for inflammatory bowel disease. Likewise, CLA-E-selectin interaction and CCL28-CCR10 and CCL17-CCR4 or CCL22-CCR4 interactions are important in T cell entry into the $\operatorname{skin}^{76}$ and therefore represent likely combinatorial targets for inflammation of the skin, such as psoriasis and atopic dermatitis. In addition, in some chronic inflammatory diseases such as rheumatoid arthritis, ecotopic lymphoid neoorganogenesis occurs locally (in the synovium) and is postulated to contribute to the perpetuation and intensity of smoldering local inflammation. Lymphotoxin-induced expression of CXCL13 and CCL21, as well as VCAM-1 and ICAM-1, is important in this process and may represent new therapeutic targets for attenuating local inflammation through the dissolution of these ectopic lymphoid aggregates ${ }^{77,78}$.

The activated macrophage is also important in many important $\mathrm{T}_{\mathrm{H}} 1$-mediated disease processes, such as multiple sclerosis, rheumatoid arthritis and atherosclerosis. Thus, modulating the trafficking patterns of macrophages may also attenuate inflammation in these chronic inflammatory diseases. Macrophage subsets are less well defined than $\mathrm{T}$ cell subsets, although studies have identified a CCR $2^{\text {hi }} \mathrm{CX} 3 \mathrm{CR} 1^{\text {lo }}$ inflammatory subset and a CCR $2^{\text {lo } C X 3 C R} 1^{\text {hi }}$ homeostatic subset ${ }^{79,80}$. Of note, preclinical studies in models of atherosclerosis ${ }^{81}$, multiple sclerosis ${ }^{82}$ and rheumatoid arthritis ${ }^{83}$ have shown that blocking CCR2 is effective in attenuating disease. Therapies aimed at blocking the interaction of CCR2 with its ligands are being evaluated in the clinic. As for T cells, combinatorial therapeutic approaches using antibodies to chemokines, integrins and selectins should be considered.

Allergic inflammation is characterized by the tissue infiltration of $\mathrm{T}_{\mathrm{H}} 2$ cells, eosinophils and mast cells secreting interleukins 4, 5 and 13 . This type of immune response is thought to have evolved to defend the host against parasitic infections. Increasing evidence suggests that this response also underlies the pathological immune response in several allergic diseases that are now epidemic in developed countries, such as asthma, food allergies and atopic dermatitis (Fig. 3). As parasitic infections have essentially been eradicated in developed countries, inhibiting the trafficking of $\mathrm{T}_{\mathrm{H}} 2$ cells, eosinophils and mast cells into tissues should be very safe. The challenge, then, is to identify the specific adhesive and signaling molecules that control the trafficking of these immune cells. The chemokine receptor CCR3 was initially thought to be such a molecule; however, subsequent studies have shown that although it is expressed on all eosinophils it is only present on a minority of $\mathrm{T}_{\mathrm{H}} 2$ cells. CCR3-deficient mice have a profound defect in eosinophil migration, but $\mathrm{T}_{\mathrm{H}} 2$ cell trafficking seems to be intact and CCR3-deficient mice can mount a tissue-specific allergic response ${ }^{84,85}$. Thus, CCR3 blockade is a viable approach for blocking eosinophil trafficking but not $\mathrm{T}_{\mathrm{H}} 2$ cell trafficking. $\mathrm{T}_{\mathrm{H}} 2$ cell trafficking is under the control of chemoattractant receptors ${ }^{86}$ and transcription factor STAT6-inducible genes $^{87}$. Thus $\mathrm{T}_{\mathrm{H}} 1$ cells 'preferentially' use CXCR3, CXCR6 and CCR5, whereas $\mathrm{T}_{\mathrm{H}} 2$ cells 'preferentially' use CCR4, CCR8 and the lipid prostaglandin $\mathrm{D}_{2}$ chemoattractant receptor $\mathrm{CRTH} 2$ (ref. 88). It remains to be determined, however, if inhibition of these or other combinations 
of chemoattractant receptors specifically expressed on $T_{H} 1$ versus $T_{H} 2$ cells can selectively inhibit the trafficking of either $\mathrm{T}$ cell type, either on its own or in combination with integrin-blocking therapy. Further complexity is added to the system, as certain important GPCRs such as BLT1, the receptor for the potent lipid chemoattractant leukotriene $\mathrm{B}_{4}$, are expressed and are functional on effector T cells, including $\mathrm{T}_{\mathrm{H}} 1$ and $\mathrm{T}_{\mathrm{H}} 2$ cells and $\mathrm{CD} 8^{+} \mathrm{T}$ cells ${ }^{89,90}$. Another complexity of the system is the differential use of chemoattractants at different microanatomic sites in a pathological lesion. For example, in the asthmatic lung, BLT1 is important in attracting $\mathrm{T}_{\mathrm{H}} 2$ cells from the interstitium into the airway $^{89}$, whereas in Crohn disease, CCL20 is highly expressed by follicleassociated epithelium well situated to recruit CCR6 $6^{+} \mathrm{T}_{\mathrm{H}} 1$ cells into the mantle zone of gut-associated lymphoid follicles ${ }^{91,92}$.

\section{Future directions}

Although it is now apparent that inhibiting leukocyte trafficking is a very effective strategy for treating a host of inflammatory diseases, other checkpoints in leukocyte trafficking can be potential targets. For example, $\mathrm{T}$ cell exit from lymphoid tissue requires sphingosine 1-phosphate and its GPCR SIP ${ }_{1}$ (ref. 93), whereas exit from peripheral tissue requires CCR7 (refs. 94,95). Pharmacological inhibition of this pathway with drugs such as FTY720 traps T cells in lymph nodes and primary organs, leading to peripheral T cell depletion ${ }^{96,97}$. FTY720 and other $\mathrm{S}_{\mathrm{P}} \mathrm{P}_{1}$ antagonists are being evaluated as new immunosuppressants for organ transplantation. Other examples include targeting proteases secreted by leukocytes, which facilitate leukocyte movement through basement membrane and the ECM in tissue, and targeting signaling molecules that link GPCR activation to changes in integrin affinity and to directed cell migration. However, the challenge for the future is to identify the key leukocyte subset that initiates a given disease and to identify the trafficking molecule(s) that will most specifically inhibit that subset of cells while leaving most leukocytes unaffected to avoid untoward infectious complications. An unavoidable consequence of inhibiting leukocyte trafficking will be that the host is left more susceptible to certain infections. It is clear, then, that better understanding of the consequences of inhibiting the trafficking of specific leukocyte subsets is needed, and patients must be screened for latent infections and/or provided with prophylaxis against the relevant potential pathogens before therapy is initiated. The goal, then, is to minimize infectious risk by identifying the trafficking molecules whose inhibition will result in the narrowest range of cellular inhibition while still allowing the therapy to be efficacious. Furthermore, regional delivery of this inhibition (such as the lung, joint or gut) and precise targeting of pathogenic leukocyte subsets will minimize systemic immunodeficiency and improve the therapeutic 'window' of treatments aimed at dampening the inflammatory response by inhibiting leukocyte trafficking.

Note: Supplementary information is available on the Nature Immunology website.

\section{ACKNOWLEDGMENTS}

Supported by the National Institutes of Health (A.D.L. and U.H.v.A.), the Dana Foundation (A.D.L. and U.H.v.A.), the Mary K. Iacocca Faculty Fellowship (U.H.v.A.), the Roche Organ Transplant Research Foundation (A.D.L.) and the Israel Science Foundation and MAIN, the EU6 Program for Migration and Inflammation (R.A.).

\section{COMPETING INTERESTS STATEMENT}

The authors declare that they have no competing financial interests.

Published online at http://www.nature.com/natureimmunology/

Reprints and permissions information is available online at http://npg.nature.com/ reprintsandpermissions/
1. Luster, A.D. Chemokines-chemotactic cytokines that mediate inflammation. N. Eng/. J. Med. 338, 436-445 (1998).

2. Ulbrich, H., Eriksson, E.E. \& Lindbom, L. Leukocyte and endothelial cell adhesion molecules as targets for therapeutic interventions in inflammatory disease. Trends Pharmacol. Sci. 24, 640-647 (2003).

3. Yonekawa, K. \& Harlan, J.M. Targeting leukocyte integrins in human diseases. J. Leukoc. Biol. 77, 129-140 (2005).

4. Cavanagh, L.L. \& Von Andrian, U.H. Travellers in many guises: the origins and destinations of dendritic cells. Immunol. Cell Biol. 80, 448-462 (2002).

5. Banchereau, J. et al. Immunobiology of dendritic cells. Annu. Rev. Immunol. 18, 767-811 (2000).

6. Jung, S. et al. In vivo depletion of $\mathrm{CD} 11 \mathrm{c}^{+}$dendritic cells abrogates priming of $\mathrm{CD}^{+}$ T cells by exogenous cell-associated antigens. Immunity 17, 211-220 (2002).

7. von Andrian, U.H. \& Mackay, C.R. T-cell function and migration. Two sides of the same coin. N. Engl. J. Med. 343, 1020-1034 (2000).

8. Kunkel, E.J. \& Butcher, E.C. Plasma-cell homing. Nat. Rev. Immunol. 3, 822-829 (2003).

9. Xie, H., Lim, Y.C., Luscinskas, F.W. \& Lichtman, A.H. Acquisition of selectin bind ing and peripheral homing properties by $\mathrm{CD}^{+}$and $\mathrm{CD}^{+}$T cells. J. Exp. Med. 189, 1765-1776 (1999).

10. Weninger, W., Crowley, M.A., Manjunath, N. \& von Andrian, U.H. Migratory properties of naive, effector, and memory CD8+ T cells. J. Exp. Med. 194, 953-966 (2001).

11. Sallusto, F., Geginat, J. \& Lanzavecchia, A. Central memory and effector memory T cell subsets: function, generation, and maintenance. Annu. Rev. Immunol. 22, 745-763 (2004).

12. Mora, J.R et al. Reciprocal and dynamic control of CD8 T cell homing by dendritic cells from skin- and gut-associated lymphoid tissues. J. Exp. Med. 201, 303-316 (2005).

13. Calzascia, T. et al. Homing phenotypes of tumor-specific CD8 T cells are predetermined at the tumor site by crosspresenting APCs. Immunity 22, 175-184 (2005).

14. Sallusto, F., Lenig, D., Mackay, C.R. \& Lanzavecchia, A. Flexible programs of chemokine receptor expression on human polarized T helper 1 and 2 lymphocytes. J. Exp. Med. 187, 875-883 (1998).

15. Austrup, F. et al. P-and E-selectin mediate recruitment of T-helper-1 but not T-helper-2 cells into inflammed tissues. Nature 385, 81-83 (1997).

16. Campbell, J.J. et al. CCR7 expression and memory T cell diversity in humans. J. Immunol. 166, 877-884 (2001).

17. Wherry, E.J. et al. Lineage relationship and protective immunity of memory CD8 T cell subsets. Nat. Immunol. 4, 225-234 (2003).

18. McEver, R.P. Selectins: lectins that initiate cell adhesion under flow. Curr. Opin. Cell Biol. 14, 581-586 (2002).

19. Rosen, S.D. Ligands for L-selectin: homing, inflammation, and beyond. Annu. Rev. Immunol. 22, 129-156 (2004).

20. Sperandio, M. et al. P-selectin glycoprotein ligand-1 mediates L-selectin-dependent leukocyte rolling in venules. J. Exp. Med. 197, 1355-1363 (2003).

21. Hafezi-Moghadam, A., Thomas, K.L., Prorock, A.J., Huo, Y. \& Ley, K. L-selectin shedding regulates leukocyte recruitment. J. Exp. Med. 193, 863-872 (2001).

22. Carman, C.V. \& Springer, T.A. Integrin avidity regulation: are changes in affinity and conformation underemphasized? Curr. Opin. Cell Biol. 15, 547-556 (2003).

23. Rot, A. \& von Andrian, U.H. Chemokines in innate and adaptive host defense: basic chemokinese grammar for immune cells. Annu. Rev. Immunol. 22, 891-928 (2004).

24. Baltus, T., Weber, K.S., Johnson, Z., Proudfoot, A.E. \& Weber, C. Oligomerization of RANTES is required for CCR 1 -mediated arrest but not CCR5-mediated transmigration of leukocytes on inflamed endothelium. Blood 102, 1985-1988 (2003).

25. Springer, T.A. Traffic signals for lymphocyte recirculation and leukocyte emigration: The multistep paradigm. Cel/ 76, 301-314 (1994).

26. Butcher, E.C. Leukocyte-endothelial cell recognition: three (or more) steps to specificity and diversity. Cel/ 67, 1033-1036 (1991).

27. Vajkoczy, P., Laschinger, M. \& Engelhardt, B. $\alpha_{4}$-integrin mediates G-protein independent capture of encephalitogenic T cell blasts on endothelial VCAM-1 in spinal cord white matter microvessels. J. Clin. Invest. 108, 557-565 (2001).

28. Ley, K. Integration of inflammatory signals by rolling neutrophils. Immunol. Rev. 186 8-18 (2002).

29. Berlin, C. et al. $\alpha_{4}$ integrins mediate lymphocyte attachment and rolling under physi ologic flow. Cell 80, 413-422 (1995).

30. Stolen, C.M. et al. Absence of the endothelial oxidase AOC3 leads to abnormal leukocyte traffic in vivo. Immunity 22, 105-115 (2005).

31. Kinashi, T. Intracellular signalling controlling integrin activation in lymphocytes. Nat. Rev. Immunol. 5, 546-559 (2005).

32. Liu, S., Calderwood, D.A. \& Ginsberg, M.H. Integrin cytoplasmic domain-binding proteins. J. Cell Sci. 113, 3563-3571 (2000).

33. Nandi, A., Estess, P. \& Siegelman, M. Bimolecular complex between rolling and firm adhesion receptors required for cell arrest; CD44 association with VLA-4 in T cell extravasation. Immunity 20, 455-465 (2004).

34. Shamri, R. et al. Lymphocyte arrest requires instantaneous induction of an extended LFA-1 conformation mediated by endothelium-bound chemokines. Nat. Immunol. 6 497-506 (2005).

35. Shimaoka, M. \& Springer, T.A. Therapeutic antagonists and the conformational regulation of the $\beta_{2}$ integrins. Curr. Top. Med. Chem. 4, 1485-1495 (2004).

36. Kim, M., Carman, C.V., Yang, W., Salas, A. \& Springer, T.A. The primacy of affinity over clustering in regulation of adhesiveness of the integrin $\alpha_{\llcorner} \beta_{2}$. J. Cell Biol. 167, 1241-1253 (2004).

37. Weber, C. \& Springer, T.A. Neutrophil accumulation on activated, surface-adherent 
platelets in flow is mediated by interaction of Mac- 1 with fibrinogen bound to $\alpha_{\text {lib }} \beta_{3}$ and stimulated by platelet-activating factor. J. Clin. Invest. 100, 2085-2093 (1997).

38. von Hundelshausen, P. et al. RANTES deposition by platelets triggers monocyte arrest on inflamed and atherosclerotic endothelium. Circulation 103, 1772-1777 (2001).

39. Ley, K. Arrest chemokines. Microcirculation 10, 289-295 (2003).

40. Laudanna, C., Kim, J.Y., Constantin, G. \& Butcher, E. Rapid leukocyte integrin activation by chemokines. Immunol. Rev. 186, 37-46 (2002).

41. Kunkel, E.J., Dunne, J.L. \& Ley, K. Leukocyte arrest during cytokine-dependent inflammation in vivo. J. Immunol. 164, 3301-3308 (2000).

42. Atarashi, K., Hirata, T., Matsumoto, M., Kanemitsu, N. \& Miyasaka, M. Rolling of Th1 cells via P-selectin glycoprotein ligand-1 stimulates LFA-1-mediated cell binding to ICAM-1. J. Immunol. 174, 1424-1432 (2005).

43. Smith, M.L., Olson, T.S. \& Ley, K. CXCR2- and E-selectin-induced neutrophil arrest during inflammation in vivo. J. Exp. Med. 200, 935-939 (2004).

44. Huo, Y. et al. Circulating activated platelets exacerbate atherosclerosis in mice deficient in apolipoprotein E. Nat. Med. 9, 61-67 (2003).

45. Vestweber, D. Regulation of endothelial cell contacts during leukocyte extravasation. Curr. Opin. Cell Biol. 14, 587-593 (2002).

46. Shimonaka, M. et al. Rap1 translates chemokine signals to integrin activation, cell polarization, and motility across vascular endothelium under flow. J. Cell Biol. 161, 417-427 (2003)

47. Dustin, M.L., Bivona, T.G. \& Philips, M.R. Membranes as messengers in T cell adhesion signaling. Nat. Immunol. 5, 363-372 (2004).

48. Vicente-Manzanares, M. \& Sanchez-Madrid, F. Role of the cytoskeleton during leukocyte responses. Nat. Rev. Immunol. 4, 110-122 (2004).

49. Wymann, M.P. \& Marone, R. Phosphoinositide 3-kinase in disease: timing, location, and scaffolding. Curr. Opin. Cell Biol. 17, 141-149 (2005).

50. Feng, D., Nagy, J.A., Pyne, K., Dvorak, H.F. \& Dvorak, A.M. Neutrophils emigrate from venules by a transendothelial cell pathway in response to fMLP. J. Exp. Med. 187, 903-915 (1998).

51. Barreiro, O. et al. Dynamic interaction of VCAM-1 and ICAM-1 with moesin and ezrin in a novel endothelial docking structure for adherent leukocytes. J. Cell Biol. 157, 1233-1245 (2002)

52. Carman, C.V. \& Springer, T.A. A transmigratory cup in leukocyte diapedesis both through individual vascular endothelial cells and between them. J. Cell Biol. 167, 377-388 (2004).

53. Johnson-Leger, C., Aurrand-Lions, M. \& Imhof, B.A. The parting of the endothelium: miracle, or simply a junctional affair? J. Cell Sci. 113, 921-933 (2000).

54. Muller, W.A. Leukocyte-endothelial-cell interactions in leukocyte transmigration and the inflammatory response. Trends Immunol. 24, 327-334 (2003).

55. Dangerfield, J., Larbi, K.Y., Huang, M.T., Dewar, A. \& Nourshargh, S. PECAM-1 (CD31) homophilic interaction up-regulates $\alpha_{6} \beta_{1}$ on transmigrated neutrophils in vivo and plays a functional role in the ability of $\alpha_{6}$ integrins to mediate leukocyte migration through the perivascular basement membrane. J. Exp. Med. 196, 1201-1211 (2002).

56. Reedquist, K.A. et al. The small GTPase, rap1, mediates CD31-induced integrin adhesion. J. Cell Biol. 148, 1151-1158 (2000).

57. Dangerfield, J.P., Wang, S. \& Nourshargh, S. Blockade of $\alpha_{6}$ integrin inhibits IL-1 $\beta$ - but not TNF- $\alpha$-induced neutrophil transmigration in vivo. J. Leukoc. Biol. 77, 159-165 (2005).

58. Ray, S.J. et al. The collagen binding $\alpha_{1} \beta_{1}$ integrin VLA-1 regulates CD8 T cell-mediated immune protection against heterologous influenza infection. Immunity 20, 167-179 (2004).

9. Timpl, R. Macromolecular organization of basement membranes. Curr. Opin. Cell Biol. 8, 618-624 (1996).

60. Sixt, M. et al. Endothelial cell laminin isoforms, laminins 8 and 10 , play decisive roles in T cell recruitment across the blood-brain barrier in experimental autoimmune encephalomyelitis. J. Cell Biol. 153, 933-946 (2001).

61. Stamenkovic, I. Extracellular matrix remodelling: the role of matrix metalloproteinases. J. Pathol. 200, 448-464 (2003).

62. Rose, M.J. \& Page, C. Glycosaminoglycans and the regulation of allergic inflammation. Curr. Drug Targets Inflamm. Allergy 3, 221-225 (2004).

63. Romanic, A.M. \& Madri, J.A. The induction of $72-\mathrm{kD}$ gelatinase in T cells upon adhesion to endothelial cells is VCAM-1 dependent. J. Cell Biol. 125, 1165-1178 (1994).

64. Vorup-Jensen, T. et al. Exposure of acidic residues as a danger signal for recognition of fibrinogen and other macromolecules by integrin $\alpha_{x} \beta_{2}$. Proc. Natl. Acad. Sci. USA 102, 1614-1619 (2005).

65. Vaday, G.G. et al. Combinatorial signals by inflammatory cytokines and chemokines mediate leukocyte interactions with extracellular matrix. J. Leukoc. Biol. 69, 885-892 (2001)

66. Wang, L., Fuster, M., Sriramarao, P. \& Esko, J.D. Endothelial heparan sulfate deficiency impairs L-selectin- and chemokine-mediated neutrophil trafficking during inflammatory responses. Nat. Immunol. 6, 902-910 (2005).

67. Wolf, K., Muller, R., Borgmann, S., Brocker, E.B. \& Friedl, P. Amoeboid shape change and contact guidance: T-lymphocyte crawling through fibrillar collagen is independent of matrix remodeling by MMPs and other proteases. Blood 102, 3262-3269 (2003).

68. Kinashi, T. et al. LAD-III, a leukocyte adhesion deficiency syndrome associated with defective Rap1 activation and impaired stabilization of integrin bonds. Blood 103 1033-1036 (2004)

69. Sekido, N. et al. Prevention of lung reperfusion injury in rabbits by a monoclonal antibody against interleukin-8. Nature 365, 654-657 (1993).

70. Harada, A. et al. Essential involvement of interleukin-8 (IL-8) in acute inflammation. J. Leukoc. Biol. 56, 559-564 (1994).

71. Kaneider, N.C., Agarwal, A., Leger, A.J. \& Kuliopulos, A. Reversing systemic inflammatory response syndrome with chemokine receptor pepducins. Nat. Med. 11, 661-665 (2005).

72. Ghosh, S. et al. Natalizumab for active Crohn's disease. N. Engl. J. Med. 348, 24-32 (2003).

73. Miller, D.H. et al. A controlled trial of natalizumab for relapsing multiple sclerosis N. Engl. J. Med. 348, 15-23 (2003).

74. Feagan, B.G. et al. Treatment of ulcerative colitis with a humanized antibody to the $\alpha_{4} \beta_{7}$ integrin. N. Engl. J. Med. 352, 2499-2507 (2005).

75. Kunkel, E.J. et al. Lymphocyte CC chemokine receptor 9 and epithelial thymusexpressed chemokine (TECK) expression distinguish the small intestinal immune compartment: Epithelial expression of tissue-specific chemokines as an organizing principle in regional immunity. J. Exp. Med. 192, 761-768 (2000).

76. Reiss, Y., Proudfoot, A.E., Power, C.A., Campbell, J.J. \& Butcher, E.C. CC chemokine receptor (CCR) 4 and the CCR10 ligand cutaneous T cell-attracting chemokine (CTACK) in lymphocyte trafficking to inflamed skin. J. Exp. Med. 194, 1541-1547 (2001).

77. Cupedo, T. \& Mebius, R.E. Role of chemokines in the development of secondary and tertiary lymphoid tissues. Semin. Immunol. 15, 243-248 (2003).

78. Hjelmstrom, P. Lymphoid neogenesis: de novo formation of lymphoid tissue in chronic inflammation through expression of homing chemokines. J. Leukoc. Biol. 69, 331-339 (2001).

79. Geissmann, F., Jung, S. \& Littman, D.R. Blood monocytes consist of two principal subsets with distinct migratory properties. Immunity 19, 71-82 (2003)

80. Ancuta, P. et al. Fractalkine preferentially mediates arrest and migration of CD16 monocytes. J. Exp. Med. 197, 1701-1707 (2003).

81. Boring, L., Gosling, J., Cleary, M. \& Charo, I.F. Decreased lesion formation in CCR2-1mice reveals a role for chemokines in the initiation of atherosclerosis. Nature 394 894-897 (1998).

82. Izikson, L., Klein, R.S., Luster, A.D. \& Weiner, H.L. Targeting monocyte recruitment in CNS autoimmune disease. Clin. Immunol. 103, 125-131 (2002).

83. Gong, J.H., Ratkay, L.G., Waterfield, J.D. \& Clark-Lewis, I. An antagonist of monocyte chemoattractant protein 1 (MCP-1) inhibits arthritis in the MRL-Ipr mouse model. J. Exp. Med. 186, 131-137 (1997).

84. Humbles, A.A. et al. The murine CCR3 receptor regulates both the role of eosinophils and mast cells in allergen-induced airway inflammation and hyperresponsiveness. Proc. Natl. Acad. Sci. USA 99, 1479-1484 (2002).

85. Ma, W. et al. CCR3 is essential for skin eosinophilia and airway hyperresponsiveness in a murine model of allergic skin inflammation. J. Clin. Invest. 109, 621-628 (2002).

86. Mathew, A., Medoff, B.D., Carafone, A.D. \& Luster, A.D. Cutting edge: Th2 cell traf ficking into the allergic lung is dependent on chemoattractant receptor signaling. J. Immunol. 169, 651-655 (2002).

87. Mathew, A. et al. Signal transducer and activator of transcription 6 controls chemokine production and Thelper cell type 2 cell trafficking in allergic pulmonary inflammation. J. Exp. Med. 193, 1087-1096 (2001).

88. Luster, A.D. \& Tager, A.M. T-cell trafficking in asthma: lipid mediators grease the way. Nat. Rev. Immunol. 4, 711-724 (2004).

89. Tager, A.M. et al. Leukotriene $B_{4}$ receptor BLT1 mediates early effector T cell recruitment. Nat. Immunol. 4, 982-990 (2003).

90. Goodarzi, K., Goodarzi, M., Tager, A.M., Luster, A.D. \& von Andrian, U.H. Leukotriene $\mathrm{B}_{4}$ and BLT1 control cytotoxic effector $T$ cell recruitment to inflamed tissues. Nat. Immunol. 4, 965-973 (2003).

91. Kwon, J.H., Keates, S., Bassani, L., Mayer, L.F. \& Keates, A.C. Colonic epithelial cells are a major site of macrophage inflammatory protein $3 \alpha$ (MIP-3 $\alpha$ ) production in normal colon and inflammatory bowel disease. Gut 51, 818-826 (2002).

92. Cook, D.N. et al. CCR6 mediates dendritic cell localization, lymphocyte homeostasis, and immune responses in mucosal tissue. Immunity 12, 495-503 (2000).

93. Matloubian, M. et al. Lymphocyte egress from thymus and peripheral lymphoid organs is dependent on S1P receptor 1. Nature 427, 355-360 (2004).

94. Bromley, S.K., Thomas, S.Y. \& Luster, A.D. Chemokine receptor CCR7 guides T cell exit from peripheral tissues and entry into afferent lymphatics. Nat. Immunol. 6, 895-901 (2005).

95. Debes, G.F. et al. Chemokine receptor CCR7 required for T lymphocyte exit from peripheral tissues. Nat. Immunol. 6, 889-894 (2005).

96. Rosen, H. \& Goetzl, E.J. Sphingosine 1-phosphate and its receptors: an autocrine and paracrine network. Nat. Rev. Immunol. 5, 560-570 (2005).

97. Cyster, J.G. Chemokines, sphingosine-1-phosphate, and cell migration in secondary lymphoid organs. Annu. Rev. Immunol. 23, 127-159 (2005). 